\title{
Noninvasive fetal $R H D$ genotyping from maternal plasma in an admixed Brazilian population
}

\author{
L.C. Schmidt ${ }^{1}$, A.C.V. Cabral ${ }^{2}$, M.A. Faria ${ }^{3}$, F. Monken ${ }^{4}$, \\ E. Tarazona-Santos ${ }^{5}$ and M.L. Martins ${ }^{3}$
}

${ }^{1}$ Departamento de Imunohematologia, Fundação Hemominas, Belo Horizonte, MG, Brasil

${ }^{2}$ Centro de Medicina Fetal, Departamento de Obstetrícia e Ginecologia, Universidade Federal de Minas Gerais, Belo Horizonte, MG, Brasil ${ }^{3}$ Departamento de Pesquisa, Fundação Hemominas, Belo Horizonte, MG, Brasil ${ }^{4}$ Centro de Medicina Fetal, Departamento de Obstetrícia e Ginecologia, Universidade Federal de Minas Gerais, Belo Horizonte, MG, Brasil ${ }^{5}$ Departamento de Biologia Geral, Universidade Federal de Minas Gerais, Belo Horizonte, MG, Brasil

Corresponding author: L.C. Schmidt

E-mail: luciana.cayres@hemominas.mg.gov.br

Genet. Mol. Res. 13 (1): 799-805 (2014)

Received August 7, 2013

Accepted November 20, 2013

Published February 7, 2014

DOI http://dx.doi.org/10.4238/2014.February.7.1

\begin{abstract}
We evaluated the efficacy of noninvasive fetal Rhesus D $(R H D)$ genotyping from maternal plasma in a highly admixed population. Fifty-five blood samples from RhD-negative pregnant women from Brazil were processed for extraction of cell-free plasma DNA. Realtime PCR was performed to amplify segments of exons 5 and 7 from the RHD gene, as well as for detection of the $S R Y$ gene to confirm the presence of fetal DNA. Fetal genotyping results were compared with the $\mathrm{RhD}$ phenotype determined from newborn cord blood samples obtained at birth. Thirty-two samples were $R H D$-positive, 18 were $R H D$-negative and 5 were inconclusive due to amplification of only
\end{abstract}


one $R H D$ exon. In 43 samples, the fetal $R H D$ genotype was compared to the neonatal $\mathrm{RhD}$ phenotype, and only one result was discordant, due to false-negative serology. There was one false $S R Y$ genotyping negative result. We conclude that noninvasive fetal $R H D$ genotyping from maternal blood provides accurate results and suggests its viability as a clinical tool for the management of $\mathrm{RhD}$-negative pregnant women in an admixed population.

Key words: RhD negative; Hemolytic disease; Fetal erythroblastosis; Maternal serum; Fetal DNA

\section{INTRODUCTION}

Alloimmunization against the RhD (RH1) red cell surface antigen is the commonest cause of hemolytic disease of the fetus/newborn (HDFN) (Finning et al., 2008). In Caucasian populations, about $10 \%$ of all pregnancies involve an RhD-negative mother and an $\mathrm{RhD}$-positive fetus, placing the mother at risk of alloimmunization and the newborn at risk of HDFN (Freeman et al., 2009).

HDFN has been an important cause of fetal or neonatal death that has not been eradicated even with the introduction of $\mathrm{RhD}$ immunoprophylaxis. While the incidence of HDFN is 1 in 21,000 live births in the United Kingdom (Finning et al., 2008), this rate is about 10 times higher in Brazil, a country with a highly heterogeneous and admixed population and where HDFN prevalence is $0.08 \%$ (DATASUS, 2006, 2009).

Because HDFN may cause newborn hyperbilirubinemia and fetal hydropsy and ultimately fetal death, it is important to predict the risk of HDFN during pregnancy, ensuring a prenatal management that may include anti-D immunoprophylaxis. The risk of alloimmunization when anti-RhD prophylaxis is used after delivery is about $1 \%$, but decreases to $0.2 \%$ when antenatal prophylaxis is also adopted (Kumpel, 2006). However, antenatal prophylaxis is not routinely adopted by the public health system in Brazil, because it is too costly and not viable, considering the current lack of a noninvasive technique in Brazil that accurately predicts fetal Rh status.

The prediction of fetal RhD phenotype is beneficial and justifies the provision of antenatal anti-RhD immunoprophylaxis when appropriate. The discovery of fetal DNA in maternal plasma (Lo et al., 1997) and the development of highly sensitive techniques such as real-time PCR allow the determination of fetal genotype by noninvasive methods. The accuracy of real-time PCR tests varies depending on the gestational time at which the sample is collected and the RHD region analyzed (Bianchi and Lo, 2001; Geifman-Holtzman et al., 2006).

Additionally, the accuracy of fetal $R H D$ genotyping to determine the RhD phenotype depends on the spectrum of $R H D$ mutations, which varies in different populations, and thus, it is important to evaluate the performance of fetal $R H D$ genotyping as a prenatal test in populations with different ethnic backgrounds before its adoption in clinical practice. In this study, we evaluated the application of real-time PCR-based fetal RHD genotyping in a heterogeneous and admixed population in Minas Gerais (Brazil) (da Silva et al., 2011). We demonstrated that this test is accurate and can thus be viably implemented in public health services. 


\section{MATERIAL AND METHODS}

\section{Population}

Blood samples from $55 \mathrm{RhD}$-negative pregnant women seen in the Outpatient Clinics for High Risk Pregnancies of the Clinical Hospital of the Federal University of Minas Gerais, Brazil, were used to establish and validate the efficacy of an $R H D$ real-time PCR assay in an admixed population. Ten milliliters of maternal peripheral blood was collected from each subject in EDTA-containing tubes and processed within a few hours (at most $24 \mathrm{~h}$ ). The samples were centrifuged at $2500 \mathrm{~g}$ for $10 \mathrm{~min}$. Plasma samples were then centrifuged at $10,000 \mathrm{~g}$ for $10 \mathrm{~min}$, and the supernatants were collected in $400-\mu \mathrm{L}$ aliquots and stored at $-80^{\circ} \mathrm{C}$ until further processing. DNA was extracted from $400 \mu \mathrm{L}$ plasma using the QIAamp DNA Blood Mini kit (Qiagen, Hilden, Germany) or Nucleo Spin Plasma XS kit (Macherey-Nagel, Germany) according to manufacturer instructions.

\section{Fetal RHD genotyping}

DNA samples were amplified using real-time PCR. Primers and probes for exon 5 (EX5-F: 5'-CGCCCTCTTCTTGTGGATG-3'; EX5-R: 5'-GAACACGGCATTCTTCCTTTC-3'; EX5-P:5'-FAM-TCTGGCCAAGTTTCAACTCTGCTCTGC-MGB-3') and for exon 7 (EX7-F: 5'-CAGCTCCATCATGGGCTACAA-3'; EX7-R： 5'-AGCACCAGCAGCACAATGTAGA-3'; EX7-P: 5'-VIC-AGCTTGCTGGGTCTGCTTGGAGAGATC-MGB-3') were used to amplify fragments of $82 \mathrm{bp}$ (exon 5) and $75 \mathrm{bp}$ (exon 7) of the RHD gene. Asingle reaction was performed with a final volume of $25 \mu \mathrm{L}$ using $1 X$ Universal PCR Master Mix (Applied Biosystems, Foster City, CA, USA), $200 \mathrm{nM}$ of each primer, $100 \mathrm{nM}$ of each probe, and $5 \mu \mathrm{LDNA}$. To confirm the presence and quality of DNA in each sample, the human albumin gene was used as an internal control for PCR in a separatereaction, using200nMofeachprimer(ALB-F:5'-GCTCAACTCCCTATTGCTATCACA-3' and ALB-R: 5'-GGGCATGACAGGTTTTGCAATATTA-3') and $100 \mathrm{nM}$ probe (ALB-P: 5'-VICTTGTGGGCTGTAATCAT-MGB-3'). Reactions were performed using the ABI Prism 7300 Sequence Detector System (Applied Biosystems) with the following cycle conditions: 2 min at $50^{\circ} \mathrm{C}$ and $10 \mathrm{~min}$ at $95^{\circ} \mathrm{C}$ followed by 45 cycles of $15 \mathrm{~s}$ at $95^{\circ} \mathrm{C}$ and $1 \mathrm{~min}$ at $60^{\circ} \mathrm{C}$. For $R H D$ genotyping, individual samples were run in duplicate. If results in duplicate tests were discrepant, DNA extraction and PCR set-up were repeated. The RHD genotyping results were interpreted as positive $(\mathrm{Ct}<42)$ or negative if both replicates and both exons were positive or negative, respectively. If the replicates showed amplification only in one exon, the RHD genotyping was considered inconclusive.

\section{Fetal $S R Y$ genotyping}

To confirm the presence of fetal DNA in the case of male fetuses, PCR for the sexdetermining region-y gene (SRY) was performed in all samples using $200 \mathrm{nM}$ of each primer (SRY-F: 5'-TCCTCAAAAGAAACCGTGCAT-3' and SRY-R: 5'-AGATTAATGGTTGCTAAGGACTGGAT-3') and $100 \mathrm{nM}$ probe (SRY-P: 5'-FAM-CACCAGCAGTAACTCCCCACAACCTCTTT-MGB-3'), as described by Johnson et al. (2004), to amplify a fragment of 78 $\mathrm{bp}$. The reaction was performed under the same conditions described above. 


\section{Analysis of real-time PCR performance}

Sensitivity, specificity, accuracy of the test, negative predictive value, and positive predictive value were calculated by comparing the genotyping results with cord blood phenotype and the newborn's gender.

\section{RESULTS}

We analyzed plasma samples from $55 \mathrm{RhD}$-negative women with gestational age ranging from 12 to 29 weeks (mean $=27$ weeks). Ages varied from 20 to 42 years old (mean $=30$ years old). Twenty-eight $(51 \%)$ women were alloimmunized. The most frequent alloantibody was anti-D (79\%), followed by anti-C (43\%), which was always present with anti-D. Anti-E, anti-M, anti-Le ${ }^{\mathrm{b}}$, and anti-Dia ${ }^{\mathrm{a}}$ were also found, each in $7 \%$ of cases. Anti-K and anti-S were found in $4 \%$ of cases. Of the 39 women who had been pregnant before, $20(51 \%)$ had a history of abortion, and $8(40 \%)$ showed a negative result for irregular antibody screening. Twelve cases $(60 \%)$ were positive for irregular antibodies, and among these anti-D was present in 10 cases (83\%), anti-S in one (8.5\%), and anti-Le in another $(8.5 \%)$.

RHD genotyping by analysis of maternal plasma showed that $32(58.2 \%)$ samples were $R H D$-positive, $18(32.7 \%)$ were $R H D$-negative, and $5(9.1 \%)$ were inconclusive due to amplification of only one $R H D$ exon (with exon 7 region being amplified in four samples and only exon 5 region being amplified in one sample).

Of the 50 samples that gave conclusive $R H D$ genotyping results, only 43 were used to compare to cord blood $\mathrm{RhD}$. In five cases $(10 \%)$, information about the newborn phenotype could not be obtained, and in two cases (4\%), pregnancy was interrupted (Table 1). With one exception, all genotyping and phenotyping results agreed (97.7\%). For the discordant case, a buccal mucosa sample and a venous blood sample were collected from the newborn and analyzed by $R H D$ genotyping and serology, and the positive result was confirmed. Cord blood serology was considered a false-negative result. Therefore, the sensitivity and specificity of the fetal $R H D$ genotyping test, as well as test accuracy, and negative and positive predictive values were $100 \%$.

Table 1. Noninvasive fetal $R H D$ and $S R Y$ genotyping by analysis of DNA purified from maternal plasma in RhD-negative women.

\begin{tabular}{|c|c|c|c|c|c|c|c|c|}
\hline \multirow[t]{2}{*}{ No. of samples } & \multicolumn{2}{|c|}{ RHD genotyping* } & \multicolumn{2}{|c|}{ Cord blood phenotype } & \multicolumn{2}{|c|}{$S R Y$ genotyping } & \multicolumn{2}{|c|}{ Newborn gender } \\
\hline & Exon 5 & Exon 7 & $\mathrm{RhD}^{+}$ & $\mathrm{RhD}^{-}$ & $S R Y^{+}$ & $S R Y^{-}$ & Male & Female \\
\hline 27 & $+/+$ & $+/+$ & 26 & $1^{* *}$ & 10 & 13 & 10 & 13 \\
\hline 16 & $-/-$ & $-/-$ & 0 & 16 & 6 & $9 * *$ & 7 & 8 \\
\hline 1 & $+/+$ & $-/-$ & 1 & 0 & 1 & 0 & 1 & 0 \\
\hline 4 & $-/-$ & $+/+$ & 3 & 1 & 1 & 2 & 1 & 2 \\
\hline
\end{tabular}

*Each sample was tested in duplicate. **The discrepancy between $R H D$ genotyping and cord blood RhD phenotype was due to false-negative serology, whereas the discrepancy between $S R Y$ genotyping and newborn gender was due to false-negative PCR.

Of the 46 maternal plasma samples subjected to $S R Y$ genotyping, 18 (39.1\%) were positive and $28(60.9 \%)$ were negative. Four $(8.7 \%)$ genotyped samples could not be compared with the newborn gender and in one case $(2.2 \%)$, gender could not be determined due 
to a congenital malformation. Thus, $42 S R Y$ genotyping results were compared with the newborn's gender (Table 1). There was $97.6 \%$ concordance between $S R Y$ genotyping results and newborn gender, along with one discordant result (false negative in genotyping). The sensitivity of $S R Y$ PCR was $94.7 \%$ and the specificity was $100 \%$. Positive predictive value was $100 \%$, negative predictive value was $95.8 \%$, and accuracy was $97.6 \%$.

\section{DISCUSSION}

In this study, we investigated the accuracy of real-time PCR to predict fetal RhD phenotype using plasma from RhD-negative pregnant women seen at a Brazilian referral center for women with high-risk pregnancy. The high sensitivity achieved by the test indicates that it can be viably used as a clinical tool for the management of RhD-negative pregnant women in an admixed population.

Concerning HDFN, the management of RhD-negative pregnant women assumes that the fetus is $\mathrm{RhD}$-positive and, therefore, the pregnancy is considered to be high risk. Thus, determination of fetal $\mathrm{RhD}$ phenotype by noninvasive fetal $R H D$ genotyping during pregnancy has a strong impact on the management of $\mathrm{RhD}$-negative pregnant women (Finning et al., 2008; Minon et al., 2008; Müller et al., 2008; Daniels et al., 2009; Mannessier, 2009). The frequency of $R H D$-positive and -negative fetuses was 56.3 and $33.3 \%$, respectively, as predicted by PCR (and confirmed at birth). This finding agrees with the literature (Avent and Reid, 2000; Grootkerk-Tax et al., 2006; Finning et al., 2008). This implies that approximately $56 \%$ of RhD-negative pregnant women analyzed should receive the antenatal anti-D immunoprophylaxis, while $33 \%$ of women could be considered at no risk of HDFN and could proceed with standard prenatal care. Consequently, the demand for specialized health services and the costs associated with laboratory tests could be reduced.

The real-time PCR test performed in this study achieved $100 \%$ sensitivity, a higher rate than that obtained with $R H D$ genotyping using DNA extracted from amniotic cells (98.7\%) (Grootkerk-Tax et al., 2006). The specificity of the test was $100 \%$, as was the predictive positive value. These values are identical to those reported for invasive $R H D$ genotyping. The negative predictive value for fetal $R H D$ genotyping was $100 \%$, which is better than that normally achieved with invasive genotyping (96.9\%) (Sesarini et al., 2009).

Studies have shown that the sensitivity of noninvasive fetal $R H D$ genotyping varies from 97.3 to $100 \%$, depending on the period of pregnancy at which the maternal sample is collected, the method of purification of free DNA from plasma, the time of DNA storage, and the efficiency of the PCR assay (Minon et al., 2008; Müller et al., 2008). The majority of published data indicate that noninvasive fetal RHD genotyping is a safe test for the management of RhD-negative pregnant women. However, in cases of negative fetal RHD genotyping results, we recommend repeating the test at more advanced stages of pregnancy.

Five fetal $R H D$ genotyping results $(9.09 \%)$ obtained in this study were considered inconclusive due to the amplification of only one $R H D$ gene exon. This percentage is higher than that reported in studies performed in the UK, China, and the USA, which varied from 3.4 to $6 \%$ (Bianchi and Lo, 2001; Finning et al., 2008). This is probably due to the heterogeneous genetic profile of the Brazilian population, which raises the possibility that different variants, rare in other populations, prevent the amplification of some exons by mismatching of primers and/or probes with their targeted genomic regions (Rodrigues et al., 2002). Therefore, in ad- 
mixed populations in which a high variety of $R H D$ alleles is expected, fetal $R H D$ genotyping may lead to false-negative results and may hinder the proper management of $\mathrm{RhD}$-negative pregnant women. However, the high accuracy of the real-time PCR test obtained in the present study suggests that fetal $R H D$ genotyping can be safely used in a highly admixed population such as the Brazilian population, provided that more than one region of the RHD gene is amplified, avoiding false results due to the presence of variant alleles such as the RHD pseudogene $(R H D \Psi)$. In two cases with inconclusive $R H D$ genotyping results, the screening for the presence of $R H D$ exons 3, 4, 5, 7, 9, and $R D H \Psi$ by conventional PCR using maternal samples amplified a fragment corresponding to $R D H \Psi$ (data not shown). In one case, the newborn was $\mathrm{RhD}$-positive, whereas the newborn was RhD-negative in the other. The paternal sample was not available for testing. This indicates that even in samples in which only the exon 7 was amplified, suggesting the presence of $R D H \Psi$ and consequently an RhD-negative phenotype, fetal phenotype cannot be reliably inferred and the result should be considered inconclusive.

One of the causes of false-negative results, which represent a challenge in fetal $R H D$ genotype determination, is the low recovery rate of fetal DNA from maternal plasma. In most centers that use fetal RHD genotyping in clinical practice, methods of fetal DNA detection have not been adopted. The internal controls used to amplify specific human genes do not allow distinction between fetal DNA and maternal DNA. For male fetuses, SRY genotyping can be applied in fetal genotyping to verify fetal DNA recovery. In this study, the predictive value of fetal $S R Y$ detection was $100 \%$.

In Brazil, the risk of HDFN is high, and high frequencies (12.5\%) of primiparous pregnant women alloimmunized by the D antigen and abortions (51\%) in women who had been pregnant before were observed, indicating that immunohematology procedures are not being adequately adopted. In this context, fetal $R H D$ genotyping is a useful tool for the proper management of RhD-negative pregnant women sensitized by anti-D, allowing the determination of fetal $\mathrm{RhD}$ phenotype and consequently the prediction of fetal risk for HDFN. Even though molecular tests have been more commonly adopted in medical services, especially in developed countries or in private healthcare services, public health service managers still associate their use with high costs. However, noninvasive fetal RHD genotyping, like the test used in this study, can be performed at low cost and is therefore feasible to be implemented in public healthcare systems.

In conclusion, fetal RHD genotyping using maternal plasma can be potentially used in clinical practice. Its use in the management of RhD-negative pregnant women can optimize costs considerably with complementary laboratory tests for monitoring pregnant women at high risk of HDFN, and can reduce the demand for specialized medical services. Moreover, the results indicate that this method is suitable to be applied in admixed populations, although further studies with a larger number of samples are needed to confirm its accuracy in these populations.

\section{ACKNOWLEDGMENTS}

Research supported by Fundação Hemominas and FAPEMIG. L.C. Schmidt, M.A. Faria, and M.L. Martins received fellowships from FAPEMIG, and E. Tarazona-Santos from CNPq.

\section{REFERENCES}

Avent ND and Reid ME (2000). The Rh blood group system: a review. Blood 95: 375-387.

Bianchi DW and Lo YM (2001). Fetomaternal cellular and plasma DNA trafficking: the Yin and the Yang. Ann. N.Y. 
Acad. Sci. 945: 119-131.

da Silva MC, Zuccherato LW, Lucena FC, Soares-Souza GB, et al. (2011). Extensive admixture in Brazilian sickle cell patients: implications for the mapping of genetic modifiers. Blood 118: 4493-4495.

Daniels G, Finning K, Martin P and Massey E (2009). Noninvasive prenatal diagnosis of fetal blood group phenotypes: current practice and future prospects. Prenat. Diagn. 29: 101-107.

DATASUS (2006). Available at [http://tabnet.datasus.gov.br/cgi/tabcgi.exe?sinasc/cnv/nvuf.def]. Accessed August 30, 2011.

DATASUS (2009). Available at [http://tabnet.datasus.gov.br/cgi/tabcgi.exe?idb2009/d23.def.]. Accessed August 30, 2011.

Finning K, Martin P, Summers J, Massey E, et al. (2008). Effect of high throughput RHD typing of fetal DNA in maternal plasma on use of anti-RhD immunoglobulin in $\mathrm{RhD}$ negative pregnant women: prospective feasibility study. $B M J$ 336: 816-818.

Freeman K, Szczepura A and Osipenko L (2009). Non-invasive fetal RHD genotyping tests: a systematic review of the quality of reporting of diagnostic accuracy in published studies. Eur. J. Obstet. Gynecol. Reprod. Biol. 142: 91-98.

Geifman-Holtzman O, Grotegut CA and Gaughan JP (2006). Diagnostic accuracy of noninvasive fetal Rh genotyping from maternal blood -- a meta-analysis. Am. J. Obstet. Gynecol. 195: 1163-1173.

Grootkerk-Tax MG, Soussan AA, de Haas M, Maaskant-van Wijk PA, et al. (2006). Evaluation of prenatal RHD typing strategies on cell-free fetal DNA from maternal plasma. Transfusion 46: 2142-2148.

Johnson KL, Dukes KA, Vidaver J, LeShane ES, et al. (2004). Interlaboratory Comparison of Fetal Male DNA detection from Commom Maternal Plasma samples by Real-Time PCR. Clin. Chem. 50: 516-521.

Kumpel BM (2006). On the immunologic basis of Rh immune globulin (anti-D) prophylaxis. Transfusion 46: 1652-1656.

Lo YM, Corbetta N, Chamberlain PF, Rai V, et al. (1997). Presence of fetal DNA in maternal plasma and serum. Lancet 350: $485-487$.

Mannessier L (2009). Immunohematological surveillance of the pregnant woman: new prevention policy. Transfus. Clin. Biol.16: 195-200.

Minon JM, Gerard C, Senterre JM, Schaaps JP, et al. (2008). Routine fetal RHD genotyping with maternal plasma: a fouryear experience in Belgium. Transfusion 48: 373-381.

Müller SP, Bartels I, Stein W, Emons G, et al. (2008). The determination of the fetal D status from maternal plasma for decision making on Rh prophylaxis is feasible. Transfusion 48: 2292-2301.

Rodrigues A, Rios M, Pellegrino J Jr, Costa FF, et al. (2002). Presence of the RHD pseudogene and the hybrid RHD-CE$\mathrm{D}(\mathrm{s})$ gene in Brazilians with the D-negative phenotype. Braz J. Med. Biol. Res. 35: 767-773.

Sesarini C, Giménez ML, Redal MA, Izbizky G, et al. (2009). Non invasive prenatal genetic diagnosis of fetal RhD and sex through the analysis of free fetal DNA in maternal plasma. Arch. Argent. Pediatr. 107: 405-409. 